\title{
Evaluation of AB - DTPA Extractant for Multinutrients Extraction in Soils
}

\author{
P. Malathi* and P. Stalin \\ Department of Soil Science and Agricultural Chemistry, Tamil Nadu Agricultural University, \\ Coimbatore - 3, Tamil Nadu, India \\ *Corresponding author
}

\begin{tabular}{|l|}
\hline K e y w o r d s \\
$\begin{array}{l}\text { ABDTPA, Multinutrient } \\
\text { extractant, Soil available } \\
\text { nutrients, Estimation }\end{array}$ \\
\hline Article Info \\
\hline $\begin{array}{l}\text { Accepted: } \\
\text { 10 February } 2018 \\
\text { Available Online: } \\
\text { 10 March } 2018\end{array}$ \\
\hline
\end{tabular}

\section{Introduction}

Escalating price of fertilizers and their effect on environment increased the need for the efficient use of fertilizers. For the diagnosis of the nutrient status of soil and deciding the need of fertilizer application, chemical methods of soil testing are widely used. Accurate determination of available nutrients by soil testing methods will pave the way for $\mathrm{Mn}, \mathrm{Zn}$ and $\mathrm{Cu}$ in alkaline soils.

\section{A B S T R A C T}

To evaluate ABDTPA, a multi nutrient extractant for simultaneous assessment of available $\mathrm{P}, \mathrm{K}, \mathrm{S}, \mathrm{Fe}, \mathrm{Mn}, \mathrm{Zn}$ and $\mathrm{Cu}, 50$ soil samples with wide range of $\mathrm{pH}$ were collected and analysed with ABDTPA and established standard methods. A pot culture experiment with maize crop was conducted to correlate the nutrients extracted by extractants with plant uptake. Correlation and regression analysis were carried out separately for each nutrient for acid, alkaline, neutral and all soils to obtain the relationship between amount of nutrients extracted by different methods and also with plant uptake. The results revealed that ABDTPA extractant is suitable for determination of available $\mathrm{P}$ in neutral $(\mathrm{r}=0.946 * * *$ with Olsen-P) and alkaline soils ( $\mathrm{r}=0.607 * * *$ with Olsen-P) than acid soils $\left(\mathrm{r}=0.450^{\mathrm{NS}}\right.$ with Bray-P). Regarding available S, ABDTPA extractant is suitable only for alkaline soils ( $\mathrm{r}=0.870 * * *$ with $\left.\mathrm{CaCl}_{2}-\mathrm{S}\right)$. ABDTPA extractant can be used for the determination of available $\mathrm{K}\left(\mathrm{r}=0.865 * * *\right.$ with $\left.\mathrm{NH}_{4} \mathrm{OAc}-\mathrm{K}\right)$, Fe $(\mathrm{r}=0.982 * * *$ with DTPA-Fe), Mn $(\mathrm{r}=0.832 * * *$ with DTPA-Mn), Zn ( $\mathrm{r}=0.952 * * *$ with DTPA-Zn) and $\mathrm{Cu}(\mathrm{r}=0.918 * * *$ with DTPA-Cu) content of soils in all the $\mathrm{pH}$ ranges. Further, correlation of nutrients extracted by extractants with that of plant uptake showed ABDTPA method is suitable for the determination of available $\mathrm{P}$ and $\mathrm{S}$ content of alkaline soils $\left(\mathrm{r}=0.723^{*}\right.$ and $0.739 *$ respectively) and not a reliable method for acid soils $\left(r=0.464^{\mathrm{NS}}\right.$ and -0.111 NS respectively). For $\mathrm{K}$ and micronutrients (Fe, $\mathrm{Mn}$ and $\mathrm{Zn}$ ), ABDTPA and standard methods correlated in a similar manner with plant uptake. Hence, it can be concluded that ABDTPA can be used as a multinutrient extractant for the simultaneous extraction of $\mathrm{P}, \mathrm{K}, \mathrm{S}, \mathrm{Fe}$,

precise fertilizer recommendation and increased use efficiency of fertilizers. Besides, soil testing provides information for research and enriching scientific knowledge. Several methods of chemical soil testing are currently used for determining the plant available macro, secondary and micro nutrients in soils. Most of these methods/extractants are specific for few or one of the plant nutrients. For analyzing a soil sample for all plant available 
nutrients, separate extraction with different extractants are required which increases the time and cost of analysis.

Multielement extractant that allows the simultaneous extraction of plant available macro, secondary and micro nutrients in soils will be highly useful for soil testing laboratories (Alva 1993). Single multinutrient extractant / Universal extraction reagents will increase laboratory productivity and decrease analytical cost. Universal extraction reagents are defined as a single extractant for use on a range of soils for the determination of both major elements and micronutrients. The nutrients can be estimated using a multielement analyzer such as the inductively coupled plasma emission spectrometer or atomic absorption spectrophotometer. Without the need for manipulation of the obtained extract, with one pass through the analyzer the concentration of the elements can be obtained. Therefore, the universal extraction reagents offer two advantages, multi-element determinations both by means of the extraction and the assay of the obtained extract (Jones, 1990).

The Ammonium Bicarbonate - DTPA is a multi - element soil test for alkaline soils developed by Soltanpour and Schwab (1977) and later modified by Soltanpour and Workman (1979) to omit the use of carbon black which adsorbed metal DTPA complexes. The $\mathrm{NH}_{4}{ }^{+}$ion replaces the exchangeable cations, $\mathrm{Na}, \mathrm{K}, \mathrm{Ca}$ and $\mathrm{Mg}$ as well as the trace metals. Di ethylene tri amine penta acetic acid (DTPA) will chelate cations such as $\mathrm{Zn}, \mathrm{Fe}, \mathrm{Cu}, \mathrm{Mn}, \mathrm{Pb}, \mathrm{Ni}$ and $\mathrm{Cd}$ thus provide availability or toxicity indices for these elements. During the shaking process, $\mathrm{CO}_{2}$ evolves from the open flask and the $\mathrm{pH}$ rise from its original value of 7.6 to about 8.5. The $\mathrm{Ca}$ and in some cases $\mathrm{Mg}$, are precipitated as carbonate salts, therefore, these two elements are not determinable.
Bicarbonate will change to carbonate as the $\mathrm{pH}$ raises precipitating $\mathrm{Ca}$ from labile calcium phosphates as calcium carbonate, thus bringing labile $\mathrm{P}$ into solution. The $\mathrm{HCO}_{3}{ }^{-}$ion will also desorb the sorbed $\mathrm{P}$. The high $\mathrm{pH}$ (8.5) will precipitate $\mathrm{Fe}$ and $\mathrm{Al}$ as their hydroxides and bring labile iron and aluminium phosphates into solution. Apparently, the stability of the DTPA Fe complex in the AB-DTPA extracting reagent is high enough to produce results comparable to the DTPA soil test of Lindsay and Norvell although the $\mathrm{pH}$ of AB-DTPA reaches about 8.5. Bicarbonate also can desorb sulphate, molybdate, selenate (or selenite) and arsenate. In addition $\mathrm{Ca}$ and other alkaline earth minerals of these anions can be solubilised as explained for calcium phosphate. Therefore, the $\mathrm{NH}_{4} \mathrm{HCO}_{3}$ - DTPA extracting reagent has a chemistry that is suitable for both cation and anion extraction. Species, such as nitrate and borate are water soluble and determinable in the extract (Soltanpour, 1985).

For using this multielement extractant routinely in soil testing laboratories, it must be compared to extractants calibrated in field experiments. Hence, this work was carried out to evaluate the suitability of AB-DTPA (Ammonium Bicarbonate- Diethylene Triamine Penta Acetic acid) as a multinutrient extractant for simultaneous extraction of available $\mathrm{P}, \mathrm{K}, \mathrm{S}, \mathrm{Fe}, \mathrm{Mn}, \mathrm{Zn}$ and $\mathrm{Cu}$ in soils.

\section{Materials and Methods}

Fifty georeferenced soil samples were collected from Coimbatore and Nilgiris districts of Tamil Nadu for analysis with AB DTPA and other extractants. Soil samples with wide $\mathrm{pH}$ range were collected because soil $\mathrm{pH}$ is one of the parameters which influences the nutrient availability. Soil samples were processed, passed through $2 \mathrm{~mm}$ sieve and used for analysis. Soil reaction $(\mathrm{pH})$ and electrical conductivity (EC) values of the 
soil samples were determined. ABDTPA, a multi nutrient extractant was used for analysis of $\mathrm{P}, \mathrm{K}, \mathrm{S}, \mathrm{Fe}, \mathrm{Mn}, \mathrm{Zn}$ and $\mathrm{Cu}$. The extracting solution is $1 \mathrm{M}$ ammonium bicarbonate $\left(\mathrm{NH}_{4} \mathrm{HCO}_{3}\right)$ and $0.005 \mathrm{M}$ DTPA adjusted to $\mathrm{pH}$ 7.6.

\section{Preparation of AB - DTPA extractant}

A $0.005 \mathrm{M}$ DTPA solution is obtained by adding $1.97 \mathrm{~g}$ DTPA to $800 \mathrm{ml}$ dilute water. Approximately $2 \mathrm{ml} \mathrm{1:1} \mathrm{ammonium} \mathrm{hydroxide}$ $\left(\mathrm{NH}_{4} \mathrm{OH}\right)$ is added to facilitate dissolution and to prevent effervescence when bicarbonate is added. When most of the DTPA is dissolved, $79.06 \mathrm{~g}$ ammonium bicarbonate $\left(\mathrm{NH}_{4} \mathrm{HCO}_{3}\right)$ is added and stirred gently until dissolved. The $\mathrm{pH}$ is adjusted to 7.6 with ammonium hydroxide and dilute $\mathrm{HCl}$. The solution is diluted to 1 litre volume with distilled water and used immediately.

\section{Extraction method}

Accurately $10 \mathrm{~g}$ soil $+20 \mathrm{ml}$ AB - DTPA shaken for 15 minutes, filtered and analysed in AAS for $\mathrm{Fe}, \mathrm{Mn}, \mathrm{Zn}$ and $\mathrm{Cu}$. Phosphorus and $S$ in the extract estimated colorimetrically and $\mathrm{K}$ by flame emission spectroscopy. The analysis for the above said nutrients was also performed with established standard methods for comparison with AB - DTPA. The details of the standard methods used for comparison are given below.

\section{Pot culture experiment}

The comparative efficiency of different extractants was further confirmed by carrying out a pot culture study to correlate nutrients extracted with their plant uptake. Of the 50 soils, 20 soils with wide $\mathrm{pH}$ range of 4.29 to 9.13 were used for the pot culture experiment. Among the soils selected, 8 were acidic, 2 were neutral and 10 were alkaline. The pot culture experiment was conducted in CRD with three replications. $\mathrm{N}$ alone in the form of urea solution $(25 \%$ of recommended $\mathrm{N}$ as basal and $50 \% \mathrm{~N}$ on 25 days after sowing) was applied to the maize hybrid NK 6240. The crop was irrigated with deionized water as and when required. The crop was grown up to 50 DAS and above ground parts were harvested. Harvested plants samples were analysed for $\mathrm{P}$, $\mathrm{K}, \mathrm{S}, \mathrm{Fe}, \mathrm{Mn}, \mathrm{Zn}$ and $\mathrm{Cu}$ content and nutrient uptake was calculated.

\section{Statistical analysis}

Correlation and regression analysis were carried out separately for each nutrient for acid, alkaline, neutral and all soils to obtain the relationship between amounts extracted by AB - DTPA method with that of standard method. The correlation coefficient (r) and slope of the regression equation were used to appraise the efficiency of the extractants. Correlation analysis was also carried out separately for each nutrient for acid, alkaline and all soils to obtain the relationship between the amounts extracted by AB-DTPA method and standard method with that of plant uptake.

\section{Results and Discussion}

\section{Soil reaction $(\mathrm{pH})$ and electrical conductivity (EC) (Table 1)}

Soil $\mathrm{pH}$ ranged from 3.74 to 9.13 and EC ranged from 0.03 to $1.28 \mathrm{dSm}^{-1}$. Of the 50 soil samples collected, 15 were acidic, 9 were neutral and 26 were alkaline. Soil $\mathrm{pH}$ and EC of the acid soils ranged from 3.74 to 6.44 and 0.03 to $0.23 \mathrm{dSm}^{-1}$ with the mean values of 4.80 and $0.10 \mathrm{dSm}^{-1}$ respectively. For neutral soils, $\mathrm{pH}$ and EC values ranged from 6.71 to 7.50 and 0.05 to $0.14 \mathrm{dSm}^{-1}$ with the mean values of 7.14 and $0.09 \mathrm{dSm}^{-1}$ respectively. The $\mathrm{pH}$ and EC values of alkaline soils ranged from 7.60 to 9.13 and 0.05 to $1.28 \mathrm{dSm}^{-1}$ with the mean values of 8.21 and $0.21 \mathrm{dSm}^{-1}$ respectively. 
Dry matter yield, content and uptake of nutrients by Maize plant (Tables 6 and 7)

The dry matter yield of the maize plant ranged from 2.59 to $13.15 \mathrm{~g} /$ plant. In extremely acidic and alkaline condition, marked reduction in dry matter yield was observed. The concentration of nutrients in maize plant ranged from 0.15 to $0.44 \%$ for $\mathrm{P}, 1.08$ to $4.81 \%$ for $\mathrm{K}, 490$ to $856 \mathrm{mg} \mathrm{kg}^{-1}$ for $\mathrm{S}, 32.7$ to $75.0 \mathrm{mg} \mathrm{kg}^{-1}$ for Fe, 33.1 to $324.4 \mathrm{mg} \mathrm{kg}^{-1}$ for $\mathrm{Mn}, 19.4$ to $46.9 \mathrm{mg} \mathrm{kg}^{-1}$ for $\mathrm{Zn}$. The plant uptake values varied from 7.5 to $31.1,72$ to $434,1.36$ to $9.51,0.11$ to $0.76,0.15$ to 1.97 and 0.12 to $0.38 \mathrm{mg} /$ plant for $\mathrm{P}, \mathrm{K}, \mathrm{S}, \mathrm{Fe}, \mathrm{Mn}$ and $\mathrm{Zn}$ respectively. Plant $\mathrm{Cu}$ concentrations were not in the detectable range and hence $\mathrm{Cu}$ uptake could not be determined.

\section{Soil available phosphorus}

\section{Acid soils}

For acid soils, the mean soil available phosphorus extracted by Bray method (96.5 $\mathrm{kg} \mathrm{ha}^{-1}$ ) was higher than ABDTPA method (3.67 kg ha ${ }^{-1}$ ) (Table 2). Correlation between Bray $-\mathrm{P}$ and ABDTPA - $\mathrm{P}$ for acid soils was non-significant $\quad\left(\mathrm{r}=0.450^{\mathrm{NS}}\right) \quad$ (Table 8). Contradictory to this, highly significant correlation between Bray - P and ABDTPA $\mathrm{P}$ have been reported by Madurapperuma and Kumaragamage (2008) with acidic low land rice soils. Slope of regression line between Bray $-\mathrm{P}$ and ABDTPA - $\mathrm{P}$ in acid soils was less than 1.0, indicating the lower extractability of phosphorus by ABDTPA extractant when compared to Bray extractant (Fig. 1) This is in line with the findings of Elrashidi et al., (2003) and Madurapperuma and Kumaragamage, (2008).

\section{Neutral soils}

The mean soil available phosphorus for neutral soils extracted by Olsen method (33.1 $\mathrm{kg} \mathrm{ha}^{-1}$ ) was higher than ABDTPA method (13.8 $\mathrm{kg} \mathrm{ha}^{-1}$ ) (Table 3). Highly significant correlation $\left(\mathrm{r}=0.946^{* * *}\right)$ was observed between Olsen $-\mathrm{P}$ and ABDTPA $-\mathrm{P}$ for neutral soils (Table 8). Slope of regression line between Olsen $-\mathrm{P}$ and ABDTPA $-\mathrm{P}$ in neutral soils was less than 1.0 which indicate the lower extractability of phosphorus by ABDTPA extractant when compared to Olsen extractant (Fig. 1).

\section{Alkaline soils}

In alkaline soils, the mean soil available phosphorus extracted by Olsen method (34.3 $\mathrm{kg} \mathrm{ha}^{-1}$ ) was higher than ABDTPA method $\left(14.8 \mathrm{~kg} \mathrm{ha}^{-1}\right)$ (Table 4). Highly significant correlation $(\mathrm{r}=0.607 * * *)$ was observed between Olsen - P and ABDTPA - P for alkaline soils (Table 8). Maftoun et al., (2003a) reported significant correlation between Olsen $\mathrm{P}$ and ABDTPA extractable P in calcareous soils. Slope of regression line between Olsen $-\mathrm{P}$ and ABDTPA $-\mathrm{P}$ in alkaline soils was less than 1.0, indicating the lower extractability of phosphorus by ABDTPA extractant when compared to Olsen extractant (Fig. 1). The correlation coefficient observed between $\mathrm{P}$ extracted by standard method and ABDTPA method was highly significant for neutral soils $\left(r=0.946^{* * *}\right)$ followed by alkaline soils $(\mathrm{r}=0.607 * * *)$ and it was non-significant for acid soils $(\mathrm{r}=$ $0.450^{\mathrm{NS}}$ ). However, Madurapperuma and Kumaragamage (2008) observed significant correlation between Bray $-\mathrm{P}$ and plant $\mathrm{P}$ uptake in acidic low land rice soils. $\mathrm{P}$ extracted by standard methods significantly correlated with plant uptake $\left(\mathrm{r}=0.805^{*}\right.$ for Bray method and $0.808^{* *}$ for Olsen method) (Table 9). P extracted by ABDTPA showed a poor relationship with plant uptake in acid soils $\left(r=0.464^{\mathrm{NS}}\right)$. Whereas, significant positive correlation was observed between ABDTPA-P and plant uptake in alkaline soils $\left(r=0.723^{*}\right)$. The results showed that ABDTPA 
method is suitable for the determination of available $\mathrm{P}$ content of alkaline soils and not a reliable method for acid soils.

\section{Soil available potassium}

Amount of soil available potassium determined by neutral normal ammonium acetate method (standard method) was in the range of 157 to $961 \mathrm{~kg} \mathrm{ha}^{-1}$ with a mean value of $501 \mathrm{~kg} \mathrm{ha}^{-1}$. Soil available $\mathrm{K}$ determined by ABDTPA method ranged from 71 to $539 \mathrm{~kg}$ $\mathrm{ha}^{-1}$ with a mean value of $307 \mathrm{~kg} \mathrm{ha}^{-1}$ (Table 5). Highly significant positive correlation was noticed between the $\mathrm{K}$ extracted by neutral normal ammonium acetate method (standard method) and ABDTPA method for acid ( $\mathrm{r}=$ $\left.0.866^{* * *}\right)$, neutral $\left(\mathrm{r}=0.825^{* *}\right)$, alkaline $(\mathrm{r}=$ $0.882 * * *)$ and all the soils put together $(\mathrm{r}=$ $0.865 * * *$ ) (Table 8). Similar results have been reported by Madurapperuma and Kumaragamage (2008) for acidic lowland rice soils and by Elrashidi et al., (2003) for acidic and alkaline upland soils. Slope of regression line between $1 \mathrm{~N} \mathrm{NH}_{4} \mathrm{OAC}-\mathrm{K}$ and ABDTPA $-\mathrm{K}$ was less than 1.0. This indicate the lower extractability of potassium by ABDTPA extractant when compared to neutral normal ammonium acetate (Fig. 1). ABDTPA extractant has lower extractability of potassium when compared to neutral normal ammonium acetate which was pointed out by the slope of regression line between $1 \mathrm{~N}$ $\mathrm{NH}_{4} \mathrm{OAC}-\mathrm{K}$ and ABDTPA - K (less than 1) (Fig. 1). Madurapperuma and Kumaragamage (2008) also observed similar results in acidic lowland rice soils. Highly significant correlations were observed between $\mathrm{K}$ extracted by neutral normal ammonium acetate method and plant uptake in alkaline soils $(\mathrm{r}=0.887 * *)$ and all soils put together $\left(\mathrm{r}=0.661^{* *}\right)$ (Table 9). This is in line with the findings of Madurapperuma and Kumaragamage (2008). Though positive correlation was observed between $\mathrm{NH}_{4} \mathrm{OAc}-\mathrm{K}$ and plant uptake in acid soils $\left(\mathrm{r}=0.540^{\mathrm{NS}}\right)$, it was not significant. The same trend was observed between ABDTPA-K and plant uptake $\left(\mathrm{r}=0.684 * * *, 0.766^{* *}\right.$ and $0.626^{\mathrm{NS}}$ for all soils put together, alkaline soils and acid soils respectively). This indicated that there is scope to use ABDTPA extractant instead of neutral normal ammonium acetate for available $\mathrm{K}$ estimation irrespective of soil type.

\section{Soil available sulphur}

\section{Acid soils}

For acid soils, the mean soil available sulphur extracted by ABDTPA method (100 $\mathrm{mg} \mathrm{kg}^{-1}$ ) was higher than $0.15 \% \mathrm{CaCl}_{2}\left(13.4 \mathrm{mg} \mathrm{kg}^{-1}\right)$ (Table 2 and Fig. 2). Non-significant and negative correlation $\left(\mathrm{r}=-0.051^{\mathrm{NS}}\right)$ was observed between $0.15 \% \mathrm{CaCl}_{2}-\mathrm{S}$ and ABDTPA - $\mathrm{S}$ for acid soils (Table 8).

\section{Neutral soils}

The mean soil available sulphur for neutral soils extracted by ABDTPA method (16.11 $\left.\mathrm{mg} \mathrm{kg}^{-1}\right)$ was higher than $0.15 \% \mathrm{CaCl}_{2}(13.00$ $\mathrm{mg} \mathrm{kg}^{-1}$ ) (Table 3 and Fig. 2). Non-significant was observed between $0.15 \% \mathrm{CaCl}_{2}-\mathrm{S}$ and ABDTPA - S for neutral soils $\left(r=0.016^{\mathrm{NS}}\right)$ (Table 8).

\section{Alkaline soils}

In alkaline soils, the mean soil available sulphur extracted by $0.15 \% \mathrm{CaCl}_{2}(19.0 \mathrm{mg}$ $\mathrm{kg}^{-1}$ ) was higher than ABDTPA method (15.6 $\mathrm{mg} \mathrm{kg}^{-1}$ ) (Table 4 and Fig. 2). Highly significant positive correlation $(\mathrm{r}=0.870 * * *)$ was observed between $0.15 \% \mathrm{CaCl}_{2}-\mathrm{S}$ and ABDTPA - S for alkaline soils (Table 8). Slope of regression line between $0.15 \% \mathrm{CaCl}_{2}$ $-\mathrm{S}$ and ABDTPA - $\mathrm{S}$ in alkaline soils was nearer to 1.0, showing almost same extractability of sulphur by ABDTPA extractant and $0.15 \% \mathrm{CaCl}_{2}$ in alkaline soils (Fig. 3). 


\section{Standard methods used for comparison with AB - DTPA}

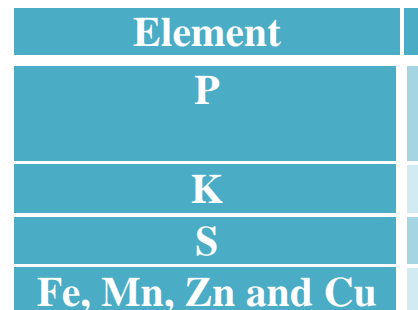

\begin{tabular}{|c|c|}
\hline Method used & Reference \\
\hline Bray method - Acid soils & Bray and Kurtz (1945) \\
Olsen method - Neutral and alkaline soils & Olsen et al., (1954) \\
\hline Neutral normal ammonium acetate method & Stanford and English (1949) \\
\hline $\mathrm{CaCl}_{2}$ extractable S & Williams and Steinbergs (1959) \\
\hline DTPA method & Lindsay and Norvell (1978) \\
\hline
\end{tabular}

Table.1 Soil reaction $(\mathrm{pH})$ and electrical conductivity

\begin{tabular}{|c|c|c|c|c|c|c|}
\hline Soil type & \multicolumn{3}{|c|}{ pH } & \multicolumn{3}{|c|}{ EC (dSm $\left.{ }^{-1}\right)$} \\
\hline & Min & Max & Mean & Min & Max & Mean \\
\hline Acid soil (n=15) & 3.74 & 6.44 & 4.80 & 0.03 & 0.23 & 0.10 \\
\hline $\begin{array}{c}\text { Neutral soil } \\
(\mathbf{n = 9})\end{array}$ & 6.71 & 7.50 & 7.14 & 0.05 & 0.14 & 0.09 \\
\hline $\begin{array}{c}\text { Alkaline soil } \\
(\mathbf{n = 2 6})\end{array}$ & 7.60 & 9.13 & 8.21 & 0.05 & 1.28 & 0.21 \\
\hline
\end{tabular}

Table.2 Range and mean of available nutrients extracted by ABDTPA and standard methods in acid soils $(n=15)$

\begin{tabular}{|c|c|c|c|c|c|c|}
\hline \multirow{2}{*}{ Nutrient } & \multicolumn{3}{|c|}{ Standard method } & \multicolumn{3}{c|}{ ABDTPA method } \\
\cline { 2 - 8 } & Min & Max & Mean & Min & Max & Mean \\
\hline P & 33.4 & 278 & 96.5 & 1.19 & 7.40 & 3.67 \\
\hline K & 171 & 867 & 545 & 115 & 514 & 306 \\
\hline S & 6.42 & 20.8 & 13.4 & 15.8 & 270 & 100 \\
\hline Fe & 31.9 & 132 & 89.3 & 24.0 & 105 & 86.0 \\
\hline $\mathbf{M n}$ & 5.06 & 159 & 49.8 & 10.7 & 181 & 43.3 \\
\hline $\mathbf{Z n}$ & 0.78 & 18.7 & 5.42 & 0.58 & 15.8 & 6.45 \\
\hline $\mathrm{Cu}$ & 0.98 & 13.0 & 3.93 & 1.72 & 16.2 & 5.85 \\
\hline
\end{tabular}

Table.3 Range and mean of available nutrients extracted by ABDTPA and standard methods in neutral soils $(n=9)$

\begin{tabular}{|c|c|c|c|c|c|c|}
\hline \multirow{2}{*}{ Nutrient } & \multicolumn{3}{|c|}{ Standard method } & \multicolumn{3}{c|}{ ABDTPA method } \\
\cline { 2 - 8 } & Min & Max & Mean & Min & Max & Mean \\
\hline P & 12.6 & 60.7 & 33.1 & 2.93 & 34.7 & 13.8 \\
\hline K & 157 & 707 & 423 & 71 & 457 & 280 \\
\hline S & 10.2 & 19.3 & 13.0 & 9.70 & 41.6 & 16.1 \\
\hline Fe & 6.38 & 31.4 & 18.3 & 8.93 & 35.2 & 21.8 \\
\hline Mn & 24.4 & 58.4 & 38.4 & 18.5 & 58.7 & 30.1 \\
\hline $\mathbf{Z n}$ & 0.81 & 8.39 & 2.82 & 1.00 & 8.62 & 2.96 \\
\hline $\mathrm{Cu}$ & 1.52 & 4.95 & 2.91 & 1.69 & 5.30 & 3.46 \\
\hline
\end{tabular}


Table.4 Range and mean of available nutrients extracted by ABDTPA and standard methods in alkaline soils $(n=26)$

\begin{tabular}{|c|c|c|c|c|c|c|}
\hline \multirow{2}{*}{ Nutrient } & \multicolumn{3}{|c|}{ Standard method } & \multicolumn{3}{c|}{ ABDTPA method } \\
& Min & Max & Mean & Min & Max & Mean \\
\hline P & 8.43 & 56.0 & 34.3 & 2.10 & 47.3 & 14.8 \\
\hline K & 178 & 961 & 503 & 118 & 539 & 317 \\
\hline S & 9.54 & 64.3 & 19.0 & 6.77 & 71.4 & 15.6 \\
\hline Fe & 3.53 & 15.8 & 7.46 & 5.38 & 23.5 & 11.5 \\
\hline Mn & 8.17 & 30.0 & 16.5 & 6.16 & 28.4 & 13.6 \\
\hline Zn & 0.49 & 3.10 & 1.14 & 0.62 & 4.68 & 1.47 \\
\hline $\mathrm{Cu}$ & 0.78 & 3.51 & 2.08 & 1.30 & 7.15 & 3.15 \\
\hline
\end{tabular}

Table.5 Range and mean of available nutrients extracted by ABDTPA and standard methods overall $(n=50)$

\begin{tabular}{|c|c|c|c|c|c|c|}
\hline \multirow{2}{*}{ Nutrient } & \multicolumn{3}{|c|}{ Standard method } & \multicolumn{3}{c|}{ ABDTPA method } \\
\hline & Min & Max & Mean & Min & Max & Mean \\
\hline K & 157 & 961 & 501 & 71 & 539 & 307 \\
\hline S & 6.42 & 64.3 & 16.2 & 6.77 & 270.8 & 41.0 \\
\hline $\mathrm{Fe}$ & 3.53 & 131.8 & 34.0 & 5.38 & 105.4 & 35.7 \\
\hline $\mathrm{Mn}$ & 5.06 & 159 & 30.5 & 6.16 & 181 & 25.5 \\
\hline $\mathrm{Zn}$ & 0.49 & 18.7 & 2.72 & 0.58 & 15.8 & 3.23 \\
\hline $\mathrm{Cu}$ & 0.78 & 13.0 & 2.79 & 1.30 & 16.2 & 4.02 \\
\hline
\end{tabular}

*- P extracted by Bray and Olsen methods based on soil $\mathrm{pH}$ and hence overall values not given

Table.6 Dry matter yield, nutrient concentration in maize plant

\begin{tabular}{|c|c|c|c|c|c|c|c|c|c|}
\hline \multirow[t]{2}{*}{ S. No. } & \multirow[t]{2}{*}{ Soil type } & \multirow[t]{2}{*}{ Soil pH } & Dry weight & $\mathbf{P}$ & $\mathbf{K}$ & $\mathbf{S}$ & $\mathrm{Fe}$ & Mn & $\mathrm{Zn}$ \\
\hline & & & g/plant & \multicolumn{2}{|c|}{$\%$} & \multicolumn{4}{|c|}{$\mathrm{mg} \mathrm{kg}^{-1}$} \\
\hline 1 & \multirow[t]{8}{*}{ Acid soil } & 4.29 & 6.63 & 0.29 & 1.25 & 787 & 43.7 & 296 & 33.1 \\
\hline 2 & & 4.43 & 2.84 & 0.26 & 3.02 & 727 & 59.5 & 324 & 45.6 \\
\hline 3 & & 4.44 & 5.72 & 0.23 & 3.53 & 655 & 40.0 & 259 & 44.2 \\
\hline 4 & & 4.77 & 5.84 & 0.23 & 3.97 & 585 & 63.2 & 230 & 45.1 \\
\hline 5 & & 4.84 & 6.60 & 0.24 & 4.81 & 628 & 51.7 & 123 & 46.5 \\
\hline 6 & & 4.94 & 11.78 & 0.44 & 3.44 & 582 & 64.3 & 103 & 24.2 \\
\hline 7 & & 5.32 & 8.59 & 0.26 & 3.67 & 812 & 75.0 & 101 & 31.0 \\
\hline 8 & & 6.44 & 6.71 & 0.21 & 1.08 & 490 & 50.4 & 38.9 & 24.6 \\
\hline 9 & \multirow[t]{2}{*}{ Neutral soil } & 6.72 & 10.02 & 0.23 & 2.57 & 717 & 60.7 & 33.2 & 24.3 \\
\hline 10 & & 7.35 & 8.91 & 0.21 & 2.44 & 566 & 41.0 & 38.2 & 20.2 \\
\hline 11 & \multirow[t]{10}{*}{ Alkaline soil } & 7.99 & 13.15 & 0.24 & 3.29 & 716 & 32.7 & 33.1 & 19.4 \\
\hline 12 & & 8.12 & 7.15 & 0.23 & 2.55 & 704 & 42.6 & 42.4 & 21.9 \\
\hline 13 & & 8.14 & 8.01 & 0.29 & 1.60 & 520 & 46.6 & 38.3 & 27.7 \\
\hline 14 & & 8.18 & 8.97 & 0.15 & 3.55 & 567 & 48.8 & 38.6 & 24.4 \\
\hline 15 & & 8.22 & 7.57 & 0.35 & 2.61 & 516 & 42.3 & 43.0 & 22.6 \\
\hline 16 & & 8.26 & 7.66 & 0.17 & 3.12 & 802 & 48.0 & 52.1 & 21.5 \\
\hline 17 & & 8.39 & 6.17 & 0.23 & 2.41 & 672 & 42.4 & 54.2 & 36.5 \\
\hline 18 & & 8.49 & 8.10 & 0.20 & 2.96 & 494 & 45.5 & 52.9 & 46.9 \\
\hline 19 & & 8.56 & 6.74 & 0.25 & 2.17 & 652 & 36.6 & 61.6 & 20.7 \\
\hline 20 & & 9.13 & 2.59 & 0.31 & 2.99 & 856 & 40.8 & 56.4 & 45.4 \\
\hline Min & & 4.29 & 2.59 & 0.15 & 1.08 & 490 & 32.7 & 33.1 & 19.4 \\
\hline Max & & 9.13 & 13.15 & 0.44 & 4.81 & 856 & 75.0 & 324 & 46.9 \\
\hline Mean & & 6.85 & 7.49 & 0.25 & 2.85 & 652 & 48.8 & 101 & 31.3 \\
\hline
\end{tabular}


Table.7 Nutrient uptake by maize plant (mg/plant)

\begin{tabular}{|c|c|c|c|c|c|c|}
\hline S. No. & P & K & S & Fe & Mn & Zn \\
\hline $\mathbf{1}$ & 19.6 & 83 & 5.21 & 0.29 & 1.97 & 0.22 \\
\hline $\mathbf{2}$ & 7.52 & 86 & 2.06 & 0.17 & 0.92 & 0.13 \\
\hline $\mathbf{3}$ & 13.2 & 202 & 3.74 & 0.23 & 1.47 & 0.25 \\
\hline $\mathbf{4}$ & 13.5 & 232 & 3.42 & 0.37 & 1.35 & 0.27 \\
\hline $\mathbf{5}$ & 16.1 & 318 & 4.14 & 0.34 & 0.81 & 0.31 \\
\hline $\mathbf{6}$ & 51.2 & 404 & 6.84 & 0.76 & 1.21 & 0.35 \\
\hline $\mathbf{7}$ & 22.5 & 316 & 6.96 & 0.65 & 0.87 & 0.27 \\
\hline $\mathbf{8}$ & 14.1 & 72 & 3.30 & 0.34 & 0.26 & 0.16 \\
\hline $\mathbf{9}$ & 23.1 & 257 & 7.18 & 0.61 & 0.33 & 0.24 \\
\hline $\mathbf{1 0}$ & 18.8 & 217 & 5.04 & 0.37 & 0.34 & 0.18 \\
\hline $\mathbf{1 1}$ & 31.1 & 434 & 9.38 & 0.43 & 0.43 & 0.25 \\
\hline $\mathbf{1 2}$ & 16.7 & 182 & 3.67 & 0.30 & 0.30 & 0.16 \\
\hline $\mathbf{1 3}$ & 22.9 & 128 & 4.15 & 0.37 & 0.31 & 0.22 \\
\hline $\mathbf{1 4}$ & 13.9 & 317 & 5.07 & 0.44 & 0.35 & 0.22 \\
\hline $\mathbf{1 5}$ & 26.6 & 198 & 3.90 & 0.32 & 0.33 & 0.17 \\
\hline $\mathbf{1 6}$ & 13.3 & 239 & 6.14 & 0.37 & 0.40 & 0.17 \\
\hline $\mathbf{1 7}$ & 14.1 & 149 & 4.15 & 0.26 & 0.33 & 0.22 \\
\hline $\mathbf{1 8}$ & 16.4 & 240 & 4.00 & 0.37 & 0.43 & 0.38 \\
\hline $\mathbf{1 9}$ & 16.6 & 146 & 4.39 & 0.25 & 0.42 & 0.14 \\
\hline $\mathbf{2 0}$ & 7.93 & 77 & 2.09 & 0.11 & 0.15 & 0.12 \\
\hline Min & $\mathbf{7 . 5 2}$ & $\mathbf{7 2}$ & $\mathbf{1 . 3 6}$ & $\mathbf{0 . 1 1}$ & $\mathbf{0 . 1 5}$ & $\mathbf{0 . 1 2}$ \\
\hline Max & $\mathbf{5 1 . 2}$ & $\mathbf{4 3 4}$ & $\mathbf{9 . 5 1}$ & $\mathbf{0 . 7 6}$ & $\mathbf{1 . 9 7}$ & $\mathbf{0 . 3 8}$ \\
\hline Mean & $\mathbf{1 9 . 0}$ & $\mathbf{2 1 5}$ & $\mathbf{4 . 6}$ & $\mathbf{0 . 3 7}$ & $\mathbf{0 . 6 5}$ & $\mathbf{0 . 2 2}$ \\
\hline
\end{tabular}

Table.8 Correlation coefficients for nutrients extracted by ABDTPA method and standard methods (Bray $-\mathrm{P}$, Olsen $-\mathrm{P}, \mathrm{NH}_{4} \mathrm{OAc}-\mathrm{K}, \mathrm{CaCl}_{2}-\mathrm{S}$ and DTPA $-\mathrm{Fe}, \mathrm{Mn}, \mathrm{Zn}$ and $\mathrm{Cu}$ )

\begin{tabular}{|c|c|c|c|c|}
\hline Elements & $\begin{array}{c}\text { All soils } \\
(\mathrm{n}=50)\end{array}$ & $\begin{array}{l}\text { Acid soils ( } \mathrm{n}=15 \\
11 \text { for Bray-P) }\end{array}$ & $\begin{array}{l}\text { Neutral soils } \\
\quad(n=9)\end{array}$ & Alkaline soils $(n=26)$ \\
\hline $\mathbf{P}$ & - & $0.450^{\mathrm{NS}}$ & $0.946 * * *$ & $0.607 * * *$ \\
\hline $\mathbf{K}$ & $0.865 * * *$ & $0.866^{* * * *}$ & $0.825^{* *}$ & $0.882 * * *$ \\
\hline $\mathbf{S}$ & $-0.055^{\mathrm{NS}}$ & $-0.051^{\mathrm{NS}}$ & $0.016^{\mathrm{NS}}$ & $0.870^{* * * *}$ \\
\hline $\mathrm{Fe}$ & $0.982 * * *$ & $0.890 * * *$ & $0.934 * * *$ & $0.778 * * *$ \\
\hline Mn & $0.832 * * *$ & $0.783 * * *$ & $0.795 *$ & $0.813 * * *$ \\
\hline $\mathrm{Zn}$ & $0.952 * * *$ & $0.950 * * *$ & $0.995 * * *$ & $0.960 * * *$ \\
\hline $\mathrm{Cu}$ & $0.918 * * *$ & $0.946 * * *$ & $0.884 * *$ & $0.761 * * *$ \\
\hline
\end{tabular}


Table.9 Correlation of nutrients extracted by different methods with plant uptake

\begin{tabular}{|c|c|c|c|c|c|c|}
\hline \multirow[t]{2}{*}{ Elements } & \multicolumn{3}{|c|}{ Standard method } & \multicolumn{3}{|c|}{ ABDTPA method } \\
\hline & $\begin{array}{c}\text { All } \\
\text { soils } \\
(\mathbf{n}=\mathbf{2 0})\end{array}$ & $\begin{array}{l}\text { Acid soils } \\
\quad(n=8)\end{array}$ & $\begin{array}{l}\text { Alkaline } \\
\text { soils } \\
(n=10)\end{array}$ & $\begin{array}{l}\text { All soils } \\
(n=20)\end{array}$ & $\begin{array}{l}\text { Acid soils } \\
\quad(n=8)\end{array}$ & $\begin{array}{l}\text { Alkaline } \\
\text { soils } \\
(n=10)\end{array}$ \\
\hline $\mathbf{P}$ & - & $0.805^{*}(\mathrm{n}=7)$ & $0.808 * *$ & - & $0.464^{\mathrm{NS}}(\mathrm{n}=7)$ & $0.723^{*}$ \\
\hline $\mathbf{K}$ & $0.661 * *$ & $0.540^{\mathrm{NS}}$ & $0.887 * *$ & $0.684 * * *$ & $0.626^{\mathrm{NS}}$ & $0.766 * *$ \\
\hline$\overline{\mathbf{S}}$ & $0.184^{\mathrm{NS}}$ & $0.511^{\mathrm{NS}}$ & $0.157^{\mathrm{NS}}$ & $-0.111^{\mathrm{NS}}$ & $-0.127^{\mathrm{NS}}$ & $0.739 *$ \\
\hline $\mathrm{Fe}$ & $0.322^{\mathrm{NS}}$ & $0.425^{\mathrm{NS}}$ & $0.777 * *$ & $0.250^{\mathrm{NS}}$ & $0.236^{\mathrm{NS}}$ & $0.670 *$ \\
\hline Mn & $0.526^{*}$ & $0.231^{\mathrm{NS}}$ & $0.296^{\mathrm{NS}}$ & $0.595^{* *}$ & $0.396^{\mathrm{NS}}$ & $0.424^{\mathrm{NS}}$ \\
\hline $\mathrm{Zn}$ & $0.583^{* *}$ & $0.595^{\mathrm{NS}}$ & $0.846^{* *}$ & $0.601 * *$ & $0.485^{\mathrm{NS}}$ & $0.883 * *$ \\
\hline
\end{tabular}

Fig.1 Relationship between soil available P and K extracted by standard methods and ABDTPA
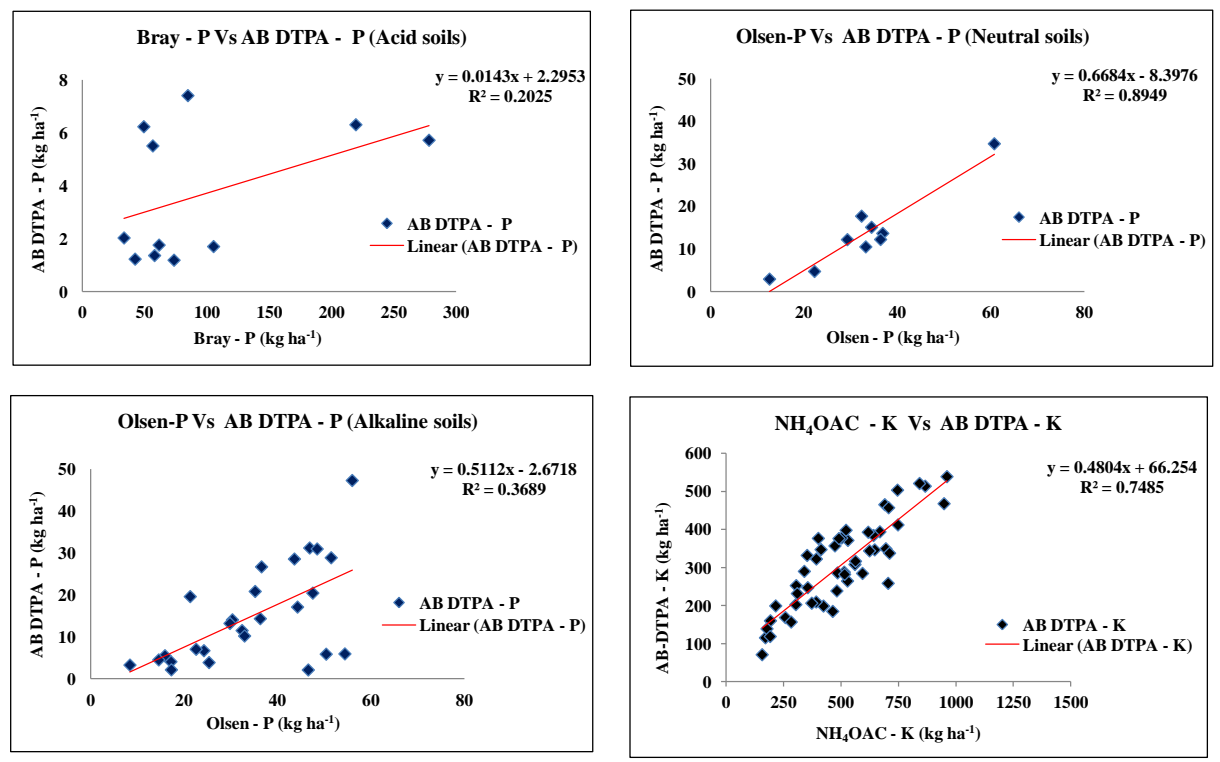

Fig.2 Relationship between soil available $\mathrm{S}$ extracted by standard method and ABDTPA
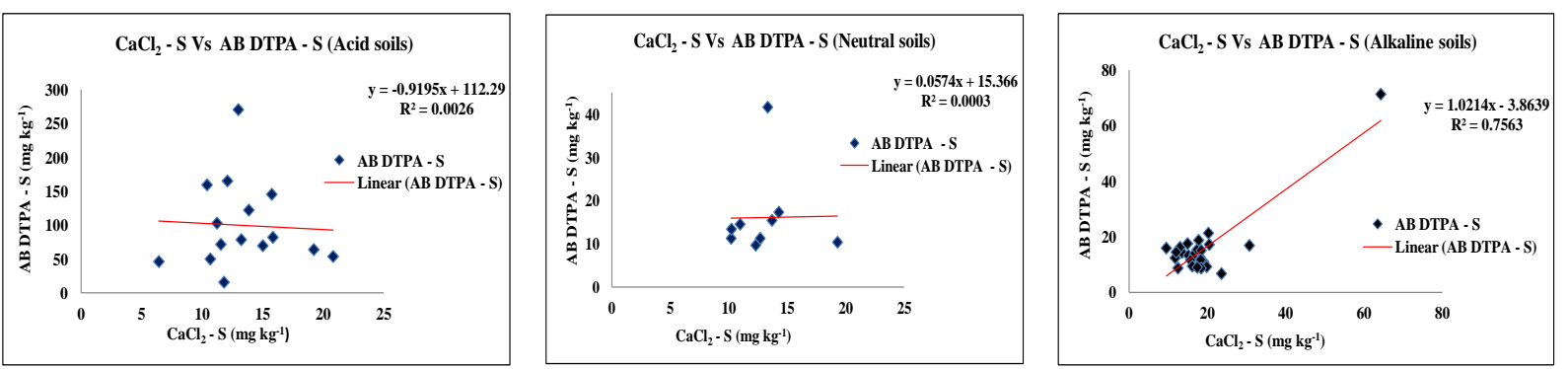
Fig.3 Relationship between soil available micronutrients extracted by standard method and ABDTPA
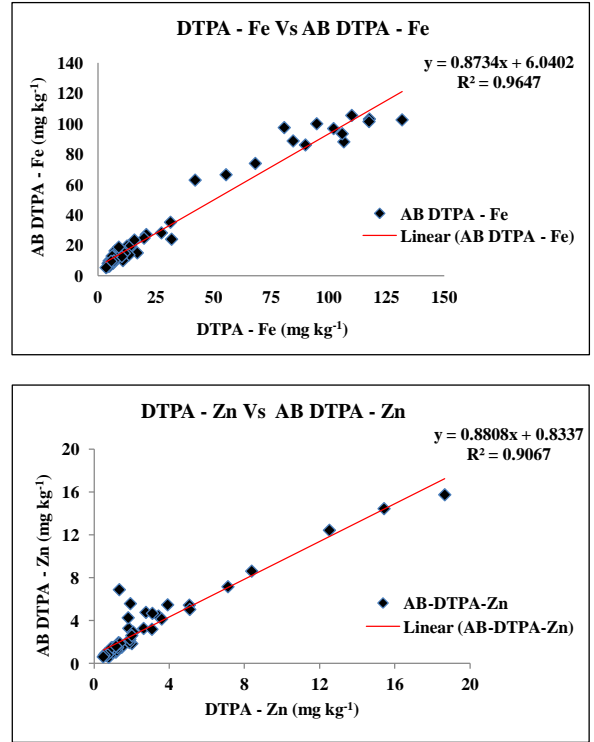

The correlation coefficient observed between $S$ extracted by standard method and ABDTPA method was positive and highly significant for alkaline soils $\left(\mathrm{r}=0.0 .870^{* * *}\right)$; not significant for neutral soils $\left(r=0.016^{\mathrm{NS}}\right)$ and negative and non-significant for acid soils $(\mathrm{r}=$ $\left.-0.051^{\mathrm{NS}}\right)($ Table 8).

Though positive correlations were observed between $0.15 \% \mathrm{CaCl}_{2}$ extractable $\mathrm{S}$ and plant uptake, they were not significant for all categories of soils $\left(\mathrm{r}=0.184^{\mathrm{NS}}, 0.157^{\mathrm{NS}}\right.$ and $0.511^{\mathrm{NS}}$ for all soils put together, alkaline soils and acid soils respectively) (Table 9). ABDTPA-S was negatively correlated with plant uptake for all soils put together $(\mathrm{r}=$ $\left.0.111^{\mathrm{NS}}\right)$ and acid soils $\left(\mathrm{r}=-0.127^{\mathrm{NS}}\right)$. Whereas, positive and significant correlation was observed between ABDTPA-S and plant uptake for alkaline soils $\left(\mathrm{r}=0.739^{*}\right)$. This showed the better performance of ABDTPA than $0.15 \% \mathrm{CaCl}_{2}$ for $\mathrm{S}$ extraction in alkaline soils. Theoretically, AB-DTPA is suitable for extraction of sulfate from soils, as most sulfate salts are soluble in aqueous solution. Furthermore, the $\mathrm{HCO}_{3}{ }^{-}$anion will desorb the
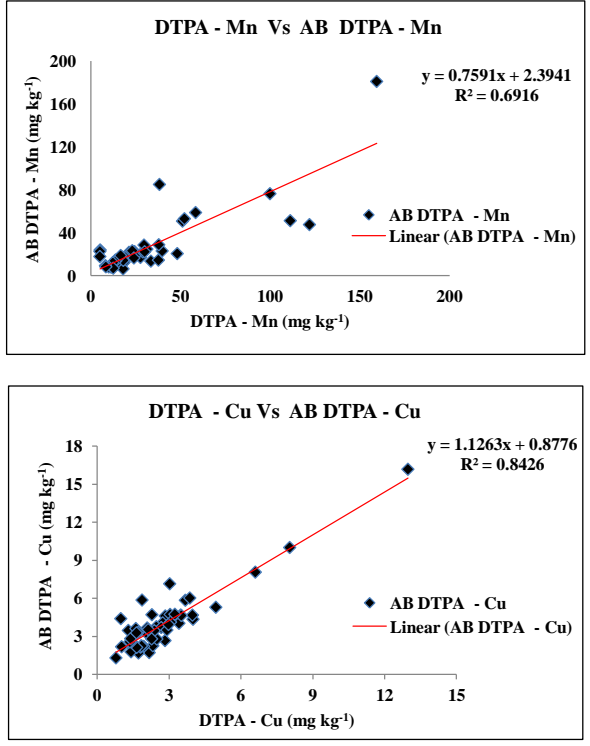

sorbed $\mathrm{SO}_{4}{ }^{-}$anion if present. Bicarbonate can also solubilize labile insoluble sulfate minerals that may be found in soils (Soltanpour 1985).

\section{Soil available iron}

Amount of soil available iron determined by DTPA (standard method) was in the range of 3.53 to $131.8 \mathrm{mg} \mathrm{kg}^{-1}$ with a mean value of $34.0 \mathrm{mg} \mathrm{kg}^{-1}$. Soil available Fe determined by ABDTPA method ranged from 5.38 to 105.4 $\mathrm{mg} \mathrm{kg}^{-1}$ with a mean value of $35.7 \mathrm{mg} \mathrm{kg}^{-1}$ (Table 5). Slope of regression line between DTPA - Fe and ABDTPA - Fe was less than 1.0, showing less extractability of $\mathrm{Fe}$ by ABDTPA extractant when compared to DTPA. Similar results have been reported by Madurapperuma and Kumaragamage (2008) for acidic lowland rice soils. Highly significant positive correlation was observed between the Fe extracted by DTPA method (standard method) and ABDTPA method for acid $\left(\mathrm{r}=0.890^{* * *}\right)$, neutral $(\mathrm{r}=0.934 * * *)$, alkaline $(\mathrm{r}=0.778 * * *)$ and all the soils put together $(\mathrm{r}=0.982 * * *)$ (Table 8$)$. 
Both DTPA and ABDTPA exhibited nonsignificant relationship with plant uptake for all soils put together $\left(\mathrm{r}=0.322^{\mathrm{NS}}\right.$ and $0.250^{\mathrm{NS}}$ for DTPA and ABDTPA respectively) and acid soils $\left(r=0.425^{\mathrm{NS}}\right.$ and $0.236^{\mathrm{NS}}$ for DTPA and ABDTPA respectively) (Table 9). Significant correlation was observed between DTPA Fe and ABDTPA Fe with plant uptake for alkaline soil $\left(\mathrm{r}=0.777^{* *}\right.$ and $0.670^{*}$ for DTPA and ABDTPA respectively). Highly significant correlation between ABDTPA extractable $\mathrm{Fe}$ and plant uptake have been reported by Al-Mustafa et al., (2001) in calcareous soils. In all types of soil, positive and highly significant correlation between $\mathrm{Fe}$ extracted by DTPA and ABDTPA methods and also similar trend between DTPA and ABDTPA extracted Fe with plant uptake were observed. This indicated that ABDTPA can be used in the place of DTPA for available Fe estimation irrespective of soil type.

\section{Soil available Manganese}

Soil available manganese determined by DTPA (standard method) was in the range of 5.06 to $159 \mathrm{mg} \mathrm{kg}^{-1}$ with a mean value of 30.5 $\mathrm{mg} \mathrm{kg}^{-1}$. Soil available $\mathrm{Mn}$ determined by ABDTPA method ranged from 6.16 to 181 $\mathrm{mg} \mathrm{kg}{ }^{-1}$ with a mean value of $25.5 \mathrm{mg} \mathrm{kg}$ (Table 5). Soil available Mn extracted by ABDTPA is lesser than DTPA extracted Fe which is indicated by the slope of the regression line (less than 1.0). Madurapperuma and Kumaragamage (2008) also reported similar findings for acidic lowland rice soils. However, Elrashidi et al., (2003) reported with acidic and alkaline soils under highland conditions, extraction of substantially higher quantities of $\mathrm{Fe}$ and $\mathrm{Mn}$ by AB DTAP than DTPA extractant. Highly significant positive correlation was observed between the Mn extracted by DTPA method (standard method) and ABDTPA method for acid $\left(\mathrm{r}=0.783^{* * *}\right)$, neutral $\left(\mathrm{r}=0.795^{*}\right)$, alkaline $\left(\mathrm{r}=0.813^{* * *}\right)$ and all the soils put together $(\mathrm{r}=0.832 * * *)$ (Table 8). For soil available $\mathrm{Mn}$ and $\mathrm{Fe}$, Madurapperuma and Kumaragamage (2008) reported similar results.

Significant correlation was observed between DTPA $\mathrm{Mn}$ and ABDTPA Mn with plant uptake for all soils put together $\left(r=0.526^{*}\right.$ and $0.595^{* *}$ for DTPA and ABDTPA respectively) (Table 9). When correlation analysis was performed for acid and alkaline soils separately, both DTPA and ABDTPA exhibited non-significant relationship with plant uptake $\left(r=0.231^{\mathrm{NS}}\right.$ and $0.396^{\mathrm{NS}}$ for DTPA and ABDTPA respectively for acid soils; $r=0.296^{\mathrm{NS}}$ and $0.424^{\mathrm{NS}}$ for DTPA and ABDTPA respectively for alkaline soils). The results are in contradiction with the findings of Madurapperuma and Kumaragamage (2008) who reported significant correlation between Mn and Fe extracted by DTPA and ABDTPA extractants with plant uptake. Mn extracted by DTPA and ABDTPA are highly correlated and both the methods correlated in a similar way with plant uptake. Hence, it is concluded that ABDTPA extractant can be used instead of DTPA for available $\mathrm{Mn}$ estimation for soils with all $\mathrm{pH}$ range.

\section{Soil available zinc}

Soil available zinc determined by DTPA (standard method) was in the range of 0.49 to $18.7 \mathrm{mg} \mathrm{kg}^{-1}$ with a mean value of $2.72 \mathrm{mg}$ $\mathrm{kg}^{-1}$. Soil available $\mathrm{Zn}$ determined by ABDTPA method ranged from 0.58 to 15.8 mg kg-1 with a mean value of $3.23 \mathrm{mg} \mathrm{kg}^{-1}$ (Table 5). The correlation observed between the $\mathrm{Zn}$ extracted by DTPA method (standard method) and ABDTPA method for acid ( $\mathrm{r}=$ $0.950 * * *)$, neutral $(\mathrm{r}=0.995 * * *)$, alkaline $(\mathrm{r}$ $=0.960 * * *)$ and all the soils put together $(\mathrm{r}=$ $0.952 * * *$ ) (Table 8 ) was positive and highly significant. Highly significant correlation between DTPA and ABDTPA extractable Zn was reported by Madurapperuma and 
Kumaragamage (2008) for acidic lowland rice soils; Elrashidi et al., (2003) for alkaline soils and by Natta Takrattanasaran et al., (2010) for calcareous soils. Highly significant correlations were observed between $\mathrm{Zn}$ extracted by DTPA and plant uptake in alkaline soils $\left(\mathrm{r}=0.846^{* *}\right)$ and all soils put together $(\mathrm{r}=0.583 * *) \quad$ (Table 9). Though positive correlation was observed between DTPA-Zn and plant uptake in acid soils $\left(r=0.595^{\mathrm{NS}}\right)$, it was not significant. The same trend was observed between ABDTPA-Zn and plant uptake $\left(\mathrm{r}=0.601 * *, 0.883^{* *}\right.$ and $0.485^{\mathrm{NS}}$ for all soils put together, alkaline soils and acid soils respectively). Similar results were observed by Abreu et al., (2002), Maftoun et al., (2003b) and Natta Takrattanasaran et al., (2010), this showed that there is a possibility to use ABDTPA extractant instead of DTPA for available $\mathrm{Zn}$ estimation irrespective of soil type.

\section{Soil available copper}

Soil available copper determined by DTPA (standard method) was in the range of 0.78 to $13.0 \mathrm{mg} \mathrm{kg}{ }^{-1}$ with a mean value of $2.79 \mathrm{mg}$ $\mathrm{kg}^{-1}$. Soil available $\mathrm{Cu}$ determined by ABDTPA method ranged from 1.30 to 16.2 $\mathrm{mg} \mathrm{kg}^{-1}$ with a mean value of $4.02 \mathrm{mg} \mathrm{kg}^{-1}$ (Table 5). Higher extractability of $\mathrm{Cu}$ by ABDTPA than DTPA has been reported by Madurapperuma and Kumaragamage (2008). Plant $\mathrm{Cu}$ concentrations were not in the detectable range. Hence, $\mathrm{Cu}$ uptake could not be determined and correlation with the extractants was not carried out. Madurapperuma and Kumaragamage (2008) also reported $\mathrm{Cu}$ in non-detectable range in rice plants. The correlation observed between the $\mathrm{Cu}$ extracted by DTPA method (standard method) and ABDTPA method for acid ( $\mathrm{r}=$ $\left.0.946^{* * *}\right)$, neutral $\left(\mathrm{r}=0.884^{* *}\right)$, alkaline $(\mathrm{r}=$ $\left.0.761^{* * *}\right)$ and all the soils put together $(\mathrm{r}=$ $0.918^{* * *}$ ) (Table 8) was positive and highly significant. Similar results have been reported by Madurapperuma and Kumaragamage (2008) in acidic lowland rice soils and Maftoun et al., (2003c) in calcareous soils. This showed that ABDTPA is a suitable extractant for the determination of available $\mathrm{Cu}$ content of soils in all the $\mathrm{pH}$ ranges.

Correlation among the nutrients extracted by ABDTPA and standard methods revealed that ABDTPA extractant is more suitable for the determination of available $P$ in neutral and alkaline soils than acid soils. Regarding available $\mathrm{S}$ estimation, ABDTPA extractant is suitable only for alkaline soils. ABDTPA extractant can be used for the determination of available $\mathrm{K}, \mathrm{Fe}, \mathrm{Mn}, \mathrm{Zn}$ and $\mathrm{Cu}$ content of soils in all the $\mathrm{pH}$ ranges. Correlation of the nutrients extracted by ABDTPA and standard methods with that of plant uptake showed that ABDTPA method is suitable for the determination of available $\mathrm{P}$ content of alkaline soils and not a reliable method for acid soils. Positive and significant correlation observed between ABDTPA-S and plant uptake for alkaline soils showed the better performance of ABDTPA than $0.15 \% \mathrm{CaCl}_{2}$ for $\mathrm{S}$ extraction in alkaline soils. It was found unsuitable for $\mathrm{S}$ estimation in acid soils. For $\mathrm{K}$ and micronutrients (Fe, $\mathrm{Mn}$ and $\mathrm{Zn}$ ) ABDTPA method can be used in the place of neutral normal ammonium acetate and DTPA extractant respectively for soils in all $\mathrm{pH}$ ranges.

The overall results indicated that ABDTPA was found suitable for the estimation of available $\mathrm{P}$ and $\mathrm{S}$ in alkaline soils, available $\mathrm{K}, \mathrm{Fe}, \mathrm{Mn}, \mathrm{Zn}$ and $\mathrm{Cu}$ in all the soils irrespective of $\mathrm{pH}$. For using as a multinutrient extractant for the simultaneous extraction of $\mathrm{P}, \mathrm{K}, \mathrm{S}, \mathrm{Fe}, \mathrm{Mn}, \mathrm{Zn}$ and $\mathrm{Cu}$, it is suitable only for alkaline soils. Hence, it can be concluded that ABDTPA can be used as a multinutrient extractant for the simultaneous extraction of $\mathrm{P}, \mathrm{K}, \mathrm{S}, \mathrm{Fe}, \mathrm{Mn}, \mathrm{Zn}$ and $\mathrm{Cu}$ in alkaline soils. 


\section{Future line of work}

To use this method for fertilizer recommendations, further evaluation of ABDTPA extractant has to be performed with more number of soils varying in texture, parent material and climate. The critical limits have to be fixed for macro and micro nutrients for prescribing fertilizer recommendations using AB-DTPA extractant. So that it can be followed in soil testing laboratories for large scale analysis of soil samples which will save the time and cost of soil analysis. Also with multinutrient extractants like ABDTPA it will be possible to make full use of the multinutrient analysers like AAS and ICP.

\section{References}

Abreu, C.A., B.V. Raij, U. Gabe, M.F. Abreu and A.P. Gonzalez. 2002. Efficiency of multinutrient extractants for determining of available zinc in soil. Commun. Soil Sci. Plant Anal. 33: 3313-3324.

Al-Mustafa, W.A., A.E. Abdallah and A.M. Falatah. 2001. Assessment of five extractants for their ability to predict iron uptake and response of sorghum grown in calcareous soils. Commun. Soil Sci. Plant Anal. 32: 907-919.

Alva A. K. 1993. comparison of Mehlich3, Mehlich1, ammonium bicarbonateDTPA, 1.0 M ammonium acetate, and $0.2 \mathrm{M}$ ammonium chloride for extraction of calcium, magnesium, phosphorus, and potassium for a wide range of soils. Commun. Soil Sci. Plant Anal. 24: 603-612.

Bray, R. H. and L. T. Kurtz. 1945. Determination of total, organic and available forms of phosphate in soils. Soil Sci. 59: 39-45.

Elrashidi, M.A., M.D. Mays and C.E. Lee. 2003. Assessment of Mehlich 3 and ammonium bicarbonate-DTPA extraction for simultaneous measurement of 15 elements in soils. Commun. Soil Sci. Plant Anal. 34: 2817-2838.

Jones J. B., Jr. 1990. Universal soil extractants: Their composition and use. Commun. Soil Sci. Plant Anal. 21 (1316): 1091-1101.

Lindsay, W. L., and W. A Norvell 1978. Development of a DTPA soil test for Zinc, iron, manganese, and copper. Soil Science Society of America Jounal. 42: $421-428$.

Madurapperuma, W.S. and D. Kumaragamage. 2008. Evaluation of ammonium bicarbonate-diethylene triamine penta acetic acid as a multinutrient extractant for acidic lowland rice soils. Commun. Soil Sci. Plant Anal. 39(11-12): 1773-1790.

Maftoun, M., H. Haghighat Nia and N. Karimian. 2003b. Evaluation of chemical extractants for predicting lowland rice response of zinc in highly calcareous soils. Commun. Soil Sci. Plant Anal. 34: 1269-1280.

Maftoun, M., M.A. Hakimzadeh Ardejkani, N. Karimian and A.M. Ronaghi. 2003a. Evaluation of phosphorus availability for paddy rice using eight chemical soil tests under oxidized and reduced soil conditions. Commun. Soil Sci. Plant Anal. 34: 2115-2129.

Maftoun, M., V. Mohasseli, N.Karimian and A.M. Ronaghi. 2003c. Laboratory and green house evaluation of five chemical extractants for estimating available copper in selected calcareous soils of Iran. Commun. Soil Sci. Plant Anal. 34(9-10): 1451-1463.

Natta Takrattanasaran, Jongruk Chanchareonsook, Suthep Thongpae and Ed Sarobol. 2010. Evaluation of Mehlich 3 and ammonium bicarbonateDTPA extractants for prediction of 
available zinc in calcareous soils in central Thailand. Kasetsart J. Nat. Sci. 44: 824-829.

Olsen, S. R., C. V. Cole, F. S. Watanabe and A. L. Dean. 1954. Estimation of available phosphorus in soil by extraction with sodium bicarbonate. Circular No.939, USDA.

Soltanpour, P. N. and S. M. Workman. 1979. Modification of the $\mathrm{NH}_{4} \mathrm{HCO}_{3}-$ DTPA soil test to omit carbon black. Commun. Soil Sci. Plant Anal. 10: 1411 - 1420.

Soltanpour, P.N. 1985. Use of Ammonium Bicarbonate DTPA soil test to evaluate elemental availability and toxicity.
Commun. Soil Sci. Plant Anal. 16: 323338.

Soltanpour, P.N. and A.P. Schwab. 1977. A new soil test for simultaneous extraction of macro and micro nutrients in alkaline soils. Commun. Soil sci. Plant Anal. 8: 195-207.

Standford, S. and L. English. 1949. Use of flame photometer in rapid soil test of $\mathrm{K}$ and Ca. Argon. J. 41: 446-447.

Williams, C. H. and A. Steinbergs. 1959. Soil sulphur fractions as chemical indices of available $\mathrm{S}$ in some Australian soils. Aust. J. Agric. Res. 10: 340-352.

\section{How to cite this article:}

Malathi, P. and Stalin, P. 2018. Evaluation of AB - DTPA Extractant for Multinutrients Extraction in Soils. Int.J.Curr.Microbiol.App.Sci. 7(03): 1192-1205. doi: https://doi.org/10.20546/ijcmas.2018.703.141 